\title{
Hereditary multiple osteochondromas in Jordanian patients: Mutational and immunohistochemical analysis of EXT1 and EXT2 genes
}

\author{
ZIYAD MOHAIDAT $^{1}$, KHALDON BODOOR $^{2}$, ROWIDA ALMOMANI $^{3}$, MOHAMMED ALORJANI $^{4}$, \\ MOHAMMAD-AKRAM AWWAD ${ }^{5}$, AUDAI BANY-KHALAF ${ }^{6}$ and KHALID AL-BATAYNEH ${ }^{7}$
}

\begin{abstract}
${ }^{1}$ Orthopedic Division, Special Surgery Department, Faculty of Medicine, Jordan University of Science and Technology, King Abdullah University Hospital, Irbid 22110; ${ }^{2}$ Department of Applied Biology, Faculty of Science; ${ }^{3}$ Department of Laboratory Medical Sciences, Faculty of Science; ${ }^{4}$ Department of Pathology, Faculty of Medicine, Jordan University of Science and Technology, Irbid 22110; ${ }^{5}$ Department of Clinical Sciences, Faculty of Medicine, Yarmouk University, Irbid 21110; ${ }^{6}$ Orthopedic Division, Special Surgery Department, Faculty of Medicine, Jordan University of Science and

Technology, Irbid 22110; ${ }^{7}$ Department of Biology, Faculty of Sciences, Yarmouk University, Irbid 21110, Jordan
\end{abstract}

Received May 28, 2020; Accepted November 26, 2020

DOI: $10.3892 / \mathrm{ol} .2020 .12412$

\begin{abstract}
The aim of the present study was to investigate the molecular characteristics of hereditary multiple osteochondromas (HMO) in a subset of Jordanian patients with a focus on the genetic variants of exostosin $(E X T 1) /(E X T 2)$ and their protein expression. Patients with HMO and their family members were included. Recorded clinical characteristics included age, sex, tumors number and location, joint deformities and associated functional limitations. Mutational analysis of EXT1 and EXT2 exonic regions was performed. Immunohistochemical staining for EXT1 and EXT2 was performed manually using two different commercially available rabbit anti-human EXT1 and EXT2 antibodies. A total of 16 patients with HMO from nine unrelated families were included, with a mean age of 13.9 years. A total of $75 \%$ $(12 / 16)$ of the patients were male and $(69 \%)(11 / 16)$ had a mild disease (class I). EXT mutation analysis revealed only EXTI gene mutations in 13 patients. Seven variants were detected, among which three were novel: c.1019G $>$ A, p. (Arg340His), c. $962+1 \mathrm{G}>\mathrm{A}$ and c.1469del, p. (Leu490Argfs*9). Of the 16 patients, 3 did not harbor any mutations for either EXTl or EXT2. Immunohistochemical examination revealed decreased expression of EXT1 protein in all patients with EXT1 mutation. Surprisingly, EXT2 protein was not detected in these patients, although none had EXT2 mutations. The majority of
\end{abstract}

Correspondence to: Dr Ziyad Mohaidat, Orthopedic Division, Special Surgery Department, Faculty of Medicine, Jordan University of Science and Technology, King Abdullah University Hospital, 1 Alramtha Street, Irbid 22110, Jordan

E-mail: zmmohaidat@just.edu.jo

Key words: hereditary multiple osteochondromas, exostosis, exostosin 1 , exostosin 2 , novel mutations
Jordanian patients with HMO, who may represent an ethnic group that is infrequently investigated, were males and had a mild clinical disease course; whereas most patients with EXT1 gene mutations were not necessarily associated with a severe clinical disease course. The role of EXT2 gene remains a subject of debate, since patients with EXT1 mutations alone did not express the non-mutated EXT2 gene.

\section{Introduction}

Hereditary multiple osteochondromas (HMO) is characterized by multiple cartilage-capped bony projections (exostoses) that usually arise from the metaphysis of long bones (1). It is also known as hereditary multiple exostoses, multiple cartilaginous exostoses, osteochondromas and diaphyseal aclasis (2). The prevalence of HMO is 1:50,000, and the male: Female ratio is 1.5:1 $(3,4)$. Although HMO can be asymptomatic and diagnosed incidentally, it can disrupt bone growth and cause short stature, unequal limb lengths and joint deformities with significant morbidity $(5,6)$. The most serious complication of $\mathrm{HMO}$ is the malignant transformation into chondrosarcoma, occurring in $0.5-5 \%$ of the patients (6). Therefore, clinical and radiological follow up is crucial for the management of patients with HMO. However, there is currently no standard follow-up protocol for HMO. Genetic analysis of EXT genes to identify patients with HMO at higher risk of developing severe disease or malignant transformation may contribute to the future management of such patients.

HMO is an autosomal dominant inherited disease with a penetrance of $100 \%$ (5). Genetic analysis of HMOs in different populations identified two main causative genes, namely exostosis 1 (EXT1) and exostosis 2 (EXT2) $(5,7,8)$. Mutations in the EXT1 and EXT2 genes account for $>90 \%$ of all HMO cases $(7,9)$. EXT1 and EXT2 both encode for a glycosyltransferase required for heparan sulfate (HS) synthesis and polymerization as HS proteoglycans (HSPGs) (8). The role of EXT1 and EXT2 proteins in HS synthesis involves the 
formation of heterocomplex of both proteins (1). HSPGs play a key role in the regulation of different signaling pathways involved in chondrocyte proliferation and differentiation in the growth plate (8).

The present study investigated 16 Jordanian index cases from nine different unrelated families with confirmed diagnosis of HMO. The different clinical characteristics in addition to the mutational spectrum of the EXT genes and the expression of their corresponding proteins were evaluated in this group of patients.

\section{Materials and methods}

Patients with HMO. The present study was conducted at several Orthopedic Surgery clinics over a 2-year period between January 2018 and December 2019. A total of 42 individuals were included in this study, among which 16 were diagnosed with HMO. Of the patients, 12 were men and 4 were women who had a mean age of 13.9 years (age range, 6-27 years). HMO diagnosis was confirmed by either histopathological or radiological examinations. These patients were evaluated clinically, and their available radiological examinations were reviewed. Disease severity was determined according to the severity score described by Mordenti et al (10) (Table I). Blood samples were collected from all participants. Paraffin-embedded tissues of patients with HMO who underwent surgical excision were available from the archives of Pathology Department. Informed consent was obtained from all individuals who participated in this study. The study protocol was approved by the Human Research Ethics Committee.

Molecular analysis. Genomic DNA from the patients and their available family members was extracted from peripheral blood samples using a Gentra Puregene kit (Qiagen $\mathrm{GmbH}$ ) following the manufacturer's instructions. The quality and concentration of the DNA was determined by NanoDrop 2000 V7.3.1 (Thermo Fisher Scientific, Inc.). All exons and exon-flanking intron sequences of EXT1 (NM_000127) and EXT2 (NM_000401) genes were amplified by PCR (polymerase chain reaction). PCR was performed in a final volume of $25 \mu \mathrm{l}$ containing $40 \mathrm{ng}$ genomic DNA, 1X Master Mix (GoTaq ${ }^{\circledR}$ Green Master Mix; Promega Corporation), and 5 pmol of forward and reverse primers (Table II). The following thermocycling conditions were used: Initial denaturing step $\left(95^{\circ} \mathrm{C}\right.$ for $7 \mathrm{~min}$ ) followed by 40 cycles of $95^{\circ} \mathrm{C}$ for $1 \mathrm{~min}$, annealing at $60^{\circ} \mathrm{C}$ for $90 \mathrm{sec}$, extension step at $72^{\circ} \mathrm{C}$ for $90 \mathrm{sec}$ and final extension at $72^{\circ} \mathrm{C}$ for $7 \mathrm{~min}$. PCR was performed using an iCycler (Bio-Rad Laboratories, Inc.). The PCR products were separated by $2 \%$ agarose gel electrophoresis, visualized by ethidium bromide and the products were purified using the Norgen's PCR Purification kit (cat. no. 45700; Norgen, Bioteck Corp.). GAPDH was used as the loading control and for normalization. Sanger sequencing was performed in both sense and antisense directions by using the BigDye Terminator Cycle Sequencing kit version 3.1 (Applied Biosystems; Thermo Fisher Scientific, Inc.), according to the manufacturer's protocol. Sequencing reactions were purified using the NucleoSEQ kit (Macherey-Nagel $\mathrm{GmbH}$ ) and final analysis performed using
Table I. HMO severity score described by Mordenti et al (10).

Class Subclass

I: No deformities-no

functional limitations
IA

IB

II: Deformities-no

functional limitations

IA

IB

III: Deformities-functional

limitations

IIIA

IIIB $\leq 5$ sites with exostosis

$>5$ sites with exostosis

$\leq 5$ sites with deformities

$>5$ sites with deformities

1 site with functional limitations

$>1$ site with functional limitations an ABI 310 DNA sequencer (Applied Biosystems; Thermo Fisher Scientific, Inc.).

The obtained sequences were compared with the normal EXT1 (NM_000127) and EXT2 (NM_000401) genes reference sequences and chromatograms were visualized by using the ChromasPro 1.34 (Technelysium Pty Ltd.) software package or Mutation Surveyor (V4.07; SoftGenetics, LLC). Sequence nomenclatures for the coding and noncoding variants were described in accordance with the Human Genome Variation Society Nomenclature standards (http://www.hgvs. org/mutnomen). To assess and predict the impact of newly identified missense variants, the Mutation taster (http://www. mutationtaster.org/) and Polyphen2 programs (http://genetics. bwh.harvard.edu/pph2/index.shtml) were used.

Immunohistochemistry. Formalin fixed, paraffin embedded HMO tissues were used in this study for immunohistochemical staining of EXT1 and EXT2. Tissues were processed following the manufacturer's instructions. Immunostaining for EXT1 and EXT2 was performed manually on the sample sections using two different commercially available rabbit anti-human EXT1(dilution 1:200; cat. no. abx100786; Abbexa Ltd.; and cat. no. HPA044394; Sigma-Aldrich; Merck KGaA) and rabbit anti-human EXT2 (dilution 1:200; cat. no. abx03435; Abbexa Ltd.; and cat. no. SAB2108124; Sigma-Aldrich; Merck KGaA) antibodies. Tissue sections were observed under a light microscopy at a magnification of $x 40$. Tissues known to express EXT1 and EXT2 (normal femoral head cartilage) were used as positive control, and negative controls were created by omitting the primary antibody step. The scoring criteria for EXT1 and EXT2 were as follow: 0, 0-10\%; 1, 10-30\%; 2, 30-85\%; and $3,>85 \%$. The intensity of the reaction was scored as: 0 , negative; 1 , weak; 2 , moderate; and 3 , strong. The samples that were scored as 1 or more were considered as positive.

\section{Results}

Patients. A total of nine families (A-I) with 42 members were included in the present study. These families included a total of 16 patients with $\mathrm{HMO}$, and three families (B, E and F) only had one affected member (Table III). 
Table II. Primer sequences used for PCR amplification and Sanger sequencing.

\begin{tabular}{|c|c|c|c|}
\hline Gene & Exon & Forward (5'-3') & Reverse (5'-3') \\
\hline \multirow[t]{13}{*}{ EXT1 } & Exon 1 & CGAGCGCAGGAGTAAACACC & CGTTTTTTGGCCTGCATGTG \\
\hline & Exon 1 & GAGCTGAAAGTGTTGATTGG & GAGACTCTGCACCTTTGGATC \\
\hline & Exon 1 & ССТCTTTGTCCTGAGTCTGG & ССАТСССССААСТТСАСАСС \\
\hline & Exon 2 & CCCCACATTCGCAATGAGTC & GAGAGGTGATAATGTTAAACCC \\
\hline & Exon 3 & CTGATTGGAACAGCTTCTGCTG & TGAAAGTTTGGACGGGGGCAGC \\
\hline & Exon 4 & GTGCATCTCTTTGTTTTACAG & GCTGAGAGAAGTGTATAAAGG \\
\hline & Exon 5 & ССТТТССАААТАТСАТСAGG & GGCCTTTAGTTCTGTATGAC \\
\hline & Exon 6 & GAGCAAGGAGGAGTAATTTTC & ATAACAGGTAAGGAGGGCGG \\
\hline & Exon 7 & AAGAGGCTTTGGGTTGGAGG & AAGTGCCCCATGGAGAAAC \\
\hline & Exon 8 & GGGAGAATTGTCCTGAAAAC & ATCGTGCAACATGAGGTGAC \\
\hline & Exon 9 & TTAGTGGGGAGAAGGTAATG & TTCCTATTTATGCAGCAGCC \\
\hline & Exon 10 & GTCTCAGAAGTCCACTTGTC & ACGTGAGTCCTCATTACCTG \\
\hline & Exon 11 & ССТTGCACTTCTCTCATCATTATCC & GAAGAGAGAGCAGCTTGACC \\
\hline \multirow[t]{14}{*}{$E X T 2$} & Exon 1 & GCCTGAATATAAGCACCTAC & AAAAGCGGGCAGTCATTGTC \\
\hline & Exon 2 & TCAAGTGTCATTTGCCATCC & СССТTСССТTTAGTTCССТG \\
\hline & Exon 3 & GGCTTGGGGATCCTTGATAG & АСТTСТАААТСТTCAGGAGG \\
\hline & Exon 4 & АCTCTGTAAACGTTAGCTGG & AGGACCCTACCCTGTAACTG \\
\hline & Exon 5 & TCAGTGGAGGTGAAGACTGG & CATAGGCCAAGCAGCTTTGC \\
\hline & Exon 6 & GTATTGCTTGGCGTCAACCC & GTAGTAGTTCTTGAACCAGG \\
\hline & Exon 7 & GGATGTTGTTTCTGCTTGTG & ACTCAGGCATTCAGCTCCTG \\
\hline & Exon 8 & CCTGGAGTTGACTATGATAG & TTATGCTGCCCTTATCAGGC \\
\hline & Exon 9 & CATGTTTGGGTTTGCTGACG & AAATGGAGGCATGCTGTCTC \\
\hline & Exon 10 & GGATACAAGCTGATTCTCCC & GCACACCTTTTGGACTCTAC \\
\hline & Exon 11 & TGGAACATCTCCAGAATCCC & AAGCCCTCTTGGCAGGTATG \\
\hline & Exon 12 & TATGAGAGAAAGCTTGTCCC & CCAATGTGACCGCATCAATC \\
\hline & Exon 13 & CATGCAACATCTCAGCTTAC & ACTATGGCTACCAGCTGCTG \\
\hline & Exon 14 & CAGACTGTGGCTACTTGAGC & AGTAGGTCAACCTTCCACCC \\
\hline
\end{tabular}

These patients had a mean age of 13.9 years at their initial diagnosis (range, 6-27 years). A total of $75 \%(12 / 16)$ of the patients were males. The total number of tumors was 135 , with over half (58\%) being located around the knee. According to HMO severity classification by Mordenti et al (10) (Table I), most $(69 \%)(11 / 16)$ of these patients had a mild disease (class I) (Table III). Moderate (class II) and severe (class III) disease forms were recorded in $2(13 \%)$ and $3(19 \%)$ patients, respectively (Table III).

EXT1 and EXT2 genes mutational analysis. Mutational analysis of the 16 patients from nine families (A-I) and their family members for both EXT1 and EXT2 genes revealed different heterozygous mutations in only EXT1 gene. While 13 patients (77\%) from seven unrelated families harbored these EXT1 mutations, the remaining 3 patients (23\%) from two families were negative for both EXT1 and EXT2 genetic variation in the targeted sequenced regions. Seven different genetic variants were identified in the EXT1 gene. These variants consisted of; two missense variants [c.1019G >A, p. (Arg340His); and c.82T>A, p. (Phe28Ile)], two deletions variants [c.918del, p.(Lys306Asnfs*53); and c.1469del, p.(Leu490Argfs*9)], one insertion variant [c.1065_1066ins26, p.(Val356Cysfs*12)], one duplication variant [c.96dup, p.(Ser33Glufs*11)] and one splice site variant $(\mathrm{c} .962+1 \mathrm{G}>\mathrm{A})(11,12)$. Three of the seven variants: c.1019G >A, p.(Arg340His); c.962+1G>A; and c.1469del, p.(Leu490Argfs*9) were novel since they were not found in known databases such as ExAC (http://exac.broadinstitute. org/), GenomeAD (https://gnomad.broadinstitute.org) and dbSNP (https://www.ncbi.nlm.nih.gov/snp). The [c.1019G>A, p. (Arg340His] variant was identified in two unrelated families (B-II.1, C-II.2, C-III.1 and C-III.2; Table III).

In family $\mathrm{A}$, a novel heterozygous variant c.918del, $\mathrm{p}$. (Lys306Asnfs*53) was identified in three affected patients (A-I.1, A-II.1 and A-II.3; Table III and Fig. 1). This deletion variant creates a frameshift starting at codon Lys306 and a new reading frame ends in a new stop codon at position 53 downstream of the mutation (Table III and Fig. 2A). Unaffected individuals in this family were wild type for this variant.

A second novel variant [c.96dup; p. (Ser33Glufs*11)] was detected in family D (D-I.1 and D-II.1; Table III and Fig. 2B). This one-base-pair duplication variant in exon 1 creates a frameshift starting at codon Ser33 and the new reading frame is predicated to end in a stop codon at position 11 (Table III and Fig. 2B).

A third novel heterozygous variant was identified in family F (F-II.3) which was an insertion of 26 base pairs (bps) in 


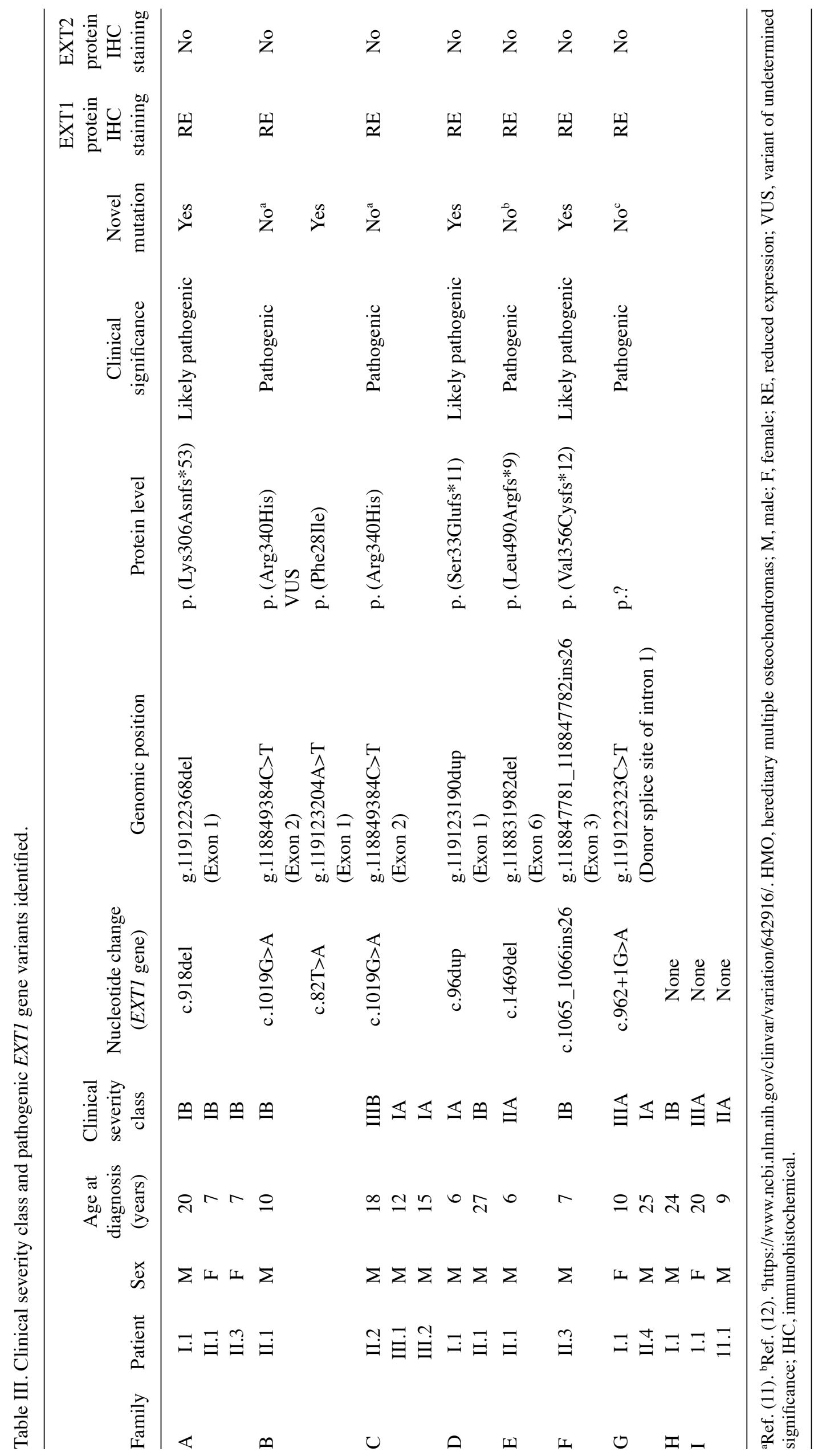


A

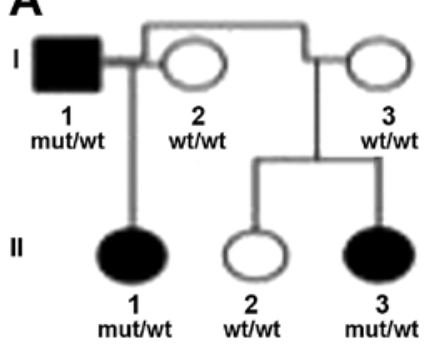

D

I

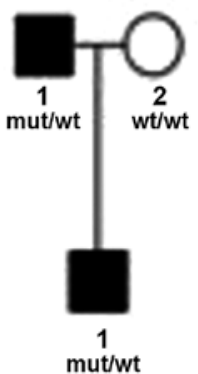

F

I

II

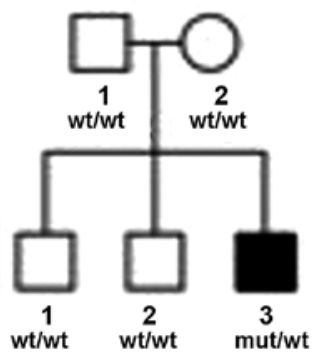

B

I

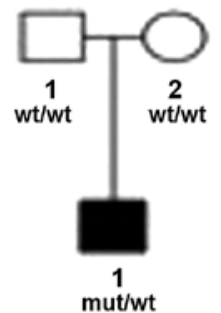

E
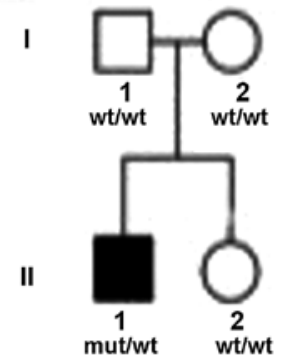

C

I

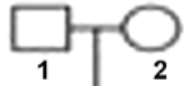

II

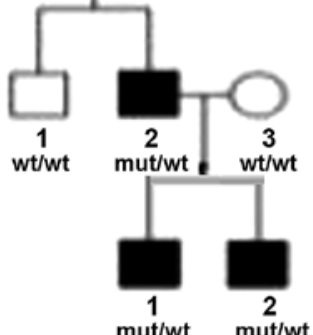

II

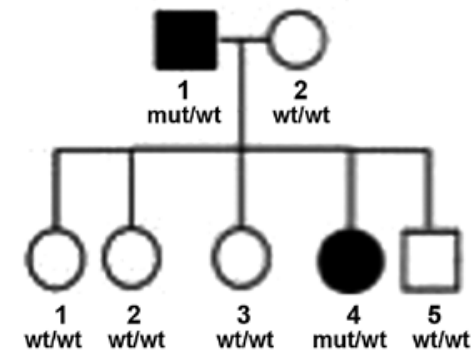

Figure 1. Pedigrees of families (A-G) with EXT1 genetic variants leading to HMO. (A) Family A, (B) Family B, (C) Family C, (D) Family D, (E) Family E, (F) Family F, (G) Family G. Squares, males; circles, females; solid symbols, affected individuals with heterozygous mutations; open symbols, unaffected individuals. EXT1 genotypes: Mu, mutant allele; wt, wild-type allele. EXT, exostosin; HMO; hereditary multiple osteochondromas.

exon 3 [c.(1065_1066ins26); p. (Val356Cysfs*12)] (Table III and Fig. 2C). This variant was predicted to create a frame shift mutation starting at codon Val356 and a new reading frame ends in a new stop codon at downstream position 12 .

Careful medical evaluation of all family members (affected and unaffected individuals) of families B, E and F, who only had one affected member each (Table III and Fig. 1) was performed. The tested mother and father of these families were clinically normal with no signs of HMO. Furthermore, genetic testing for the presence of a causative genetic variant of the parents showed negative mutation results. The affected individuals (B-II.1, E-II.1 and F-II.3) in these families carry a de novo mutation and were the first ones who gained the mutant allele.

Immunohistochemical staining of EXT1 and EXT2. Osteochondroma tissue was available for immunohistochemical staining from 12 of the 13 patients with EXT1 gene mutations. EXT1 protein expression was found to be significantly decreased (weak staining pattern; Fig. 3B) in all examined tissues, apart from tissues obtained from the 2 proband patients of family D (D-I.1 and D-II.1). Their tissues exhibited moderate staining pattern when compared to EXT1 protein expression in normal chondrocytes (Fig. 3A). A novel variant [c.96dup; p. (Ser33Glufs*11)] was detected in this family. With regards to the EXT2 gene, no protein expression was detected in any osteochondroma tissues from the 12 tested patients (Fig. 3B).

\section{Discussion}

Several HMO studies investigated the associations of the different clinical characteristics with the genetic findings in different populations. The clinical characteristics of patients with HMO in the present study, including the mean age at diagnosis of 13.9 years, as well as male predilection, were similar to what was reported in the literature $(1,13)$. The knee joint was the most common tumor location, which is also consistent with the findings of other studies (14-16).

HMO severity was reported using various clinical classifications (10). These classifications were based on the clinical parameters of HMO patients, including, age, tumor number, joint deformities, limb length discrepancy, in addition to the morbidity associated with HMO tumors. The clinical classification, used in the present study, revealed that the majority of Jordanian patients with HMO have a mild disease form (class 1). Since different HMO studies $(14,17,18)$ used different scoring systems to assess 


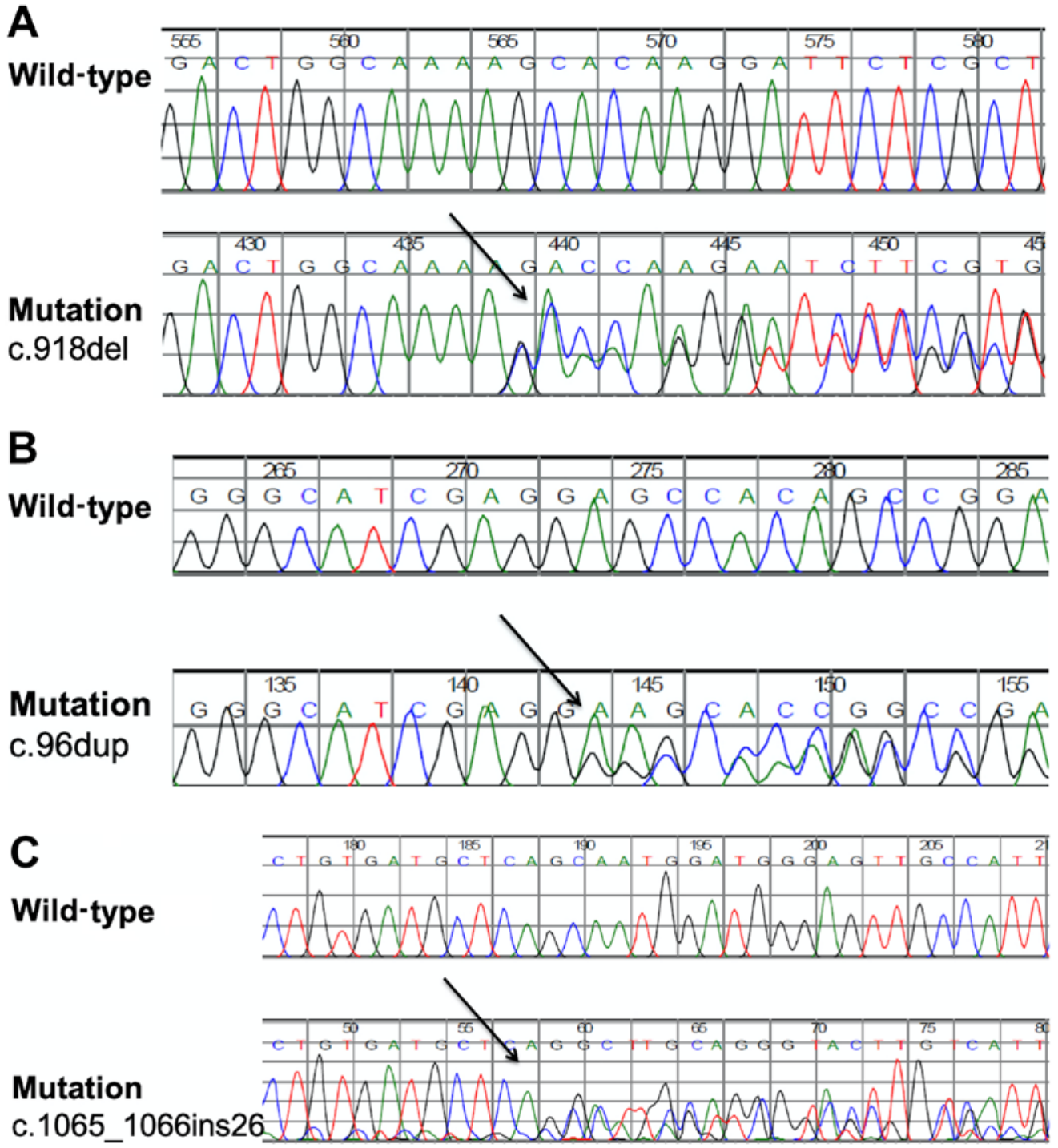

Figure 2. Sanger sequencing data of (A-C) the three novel variants identified in the present study, patients (mutation) and controls (wild-type). (A) Novel Mutation c.918del, (B) Novel Mutation c.96dup, (C) Novel Mutation c.1065_1066ins26. EXT, exostosin; HMO; hereditary multiple osteochondromas.

HMO severity, comparing HMO severity among different populations can be difficult.

HMO is not only clinically heterogenous, but it is also genetically heterogenous $(6,17,18)$. Mutational analysis studies reported variable frequencies of EXT1 and EXT2 mutations in different ethnic groups (13). Several authors reported EXT1 mutations to be more common, particularly in Caucasians (7,19-21). Although the present study investigated a different ethnic group, it revealed a similar predominance of EXT1 mutations in this group of Jordanian patients with HMO. On the other hand, no potential pathogenic genetic variants of EXT2 gene were identified in the present study. This was inconsistent with other studies which reported that EXT2 mutations to be present in $20-45 \%$ of the patients (7). This inconsistency may be explained by the presence of mutations in noncoding parts of the genes. In addition, the small number of the included patients can be a contributing factor.

Several phenotype-genotype studies of HMO reported that EXT1 gene mutations were more likely than EXT2 gene mutations to be associated with a more severe form of the disease $(14,15,18,20-23)$. Other studies reported no difference in disease severity between these two gene mutations $(19,24)$. In the present study, only 2 of the 13 patients with EXT1 mutations exhibited a severe form of the disease (class III). This can be attributed to the variability of HMO severity, even among patients with the same EXT gene mutations particularly EXT1 gene $(18,25)$. In addition, ethnicity can be considered as another influential factor. This is also consistent with the findings of a previous study investigating osteochondroma in Jordanian patients in whom a milder form of HMO was observed compared with that of other populations (4).

With regards to the mutational analysis of the patients in the present study, EXT1 allelic heterogeneity was observed and the identified mutations were shown to be dispersed throughout the coding regions of the gene (Fig. 4). Furthermore, the truncated mutations occurred in $66.6 \%$ of the tested families. These results are similar to those reported from different studies in the literature (9). A mutational database from these studies is assembled in the Multiple Osteochondromas Mutation Database (MOdb) (http://medgen.ua.ac.be/LOVDv.2.0/), with $>600$ and 200 different mutations in EXT1 and EXT2, respectively. The majority of these mutations $(80 \%)$ are nonsense, 

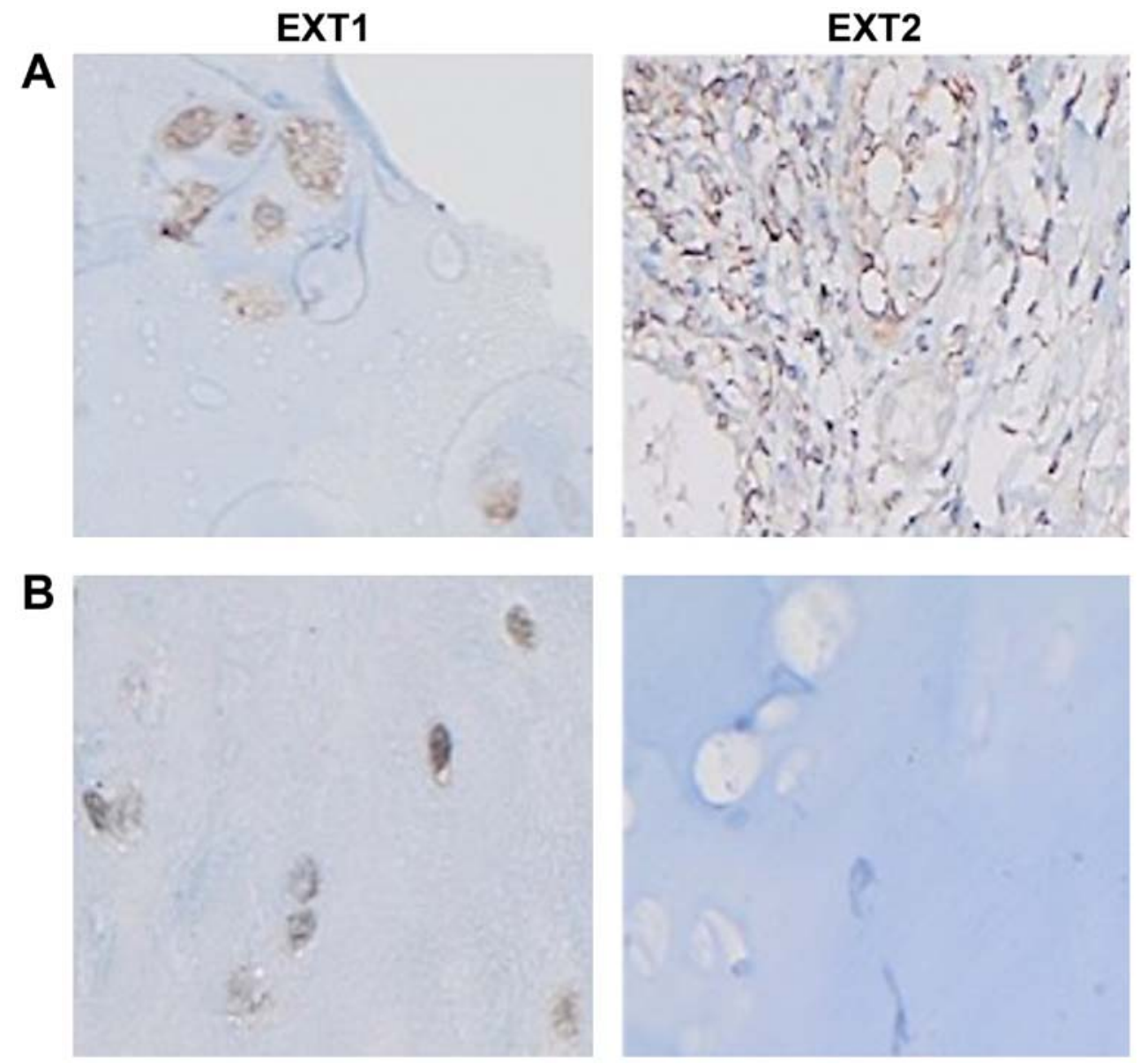

Figure 3. Immunohistochemical staining of chondrocytes. (A) Immunohistochemical staining of EXT1 and EXT2 from control unaffected tissues (magnification, x40). (B) Immunohistochemical staining of EXT1 and EXT2 from a patient with hereditary multiple osteochondromas (magnification, x40). EXT, exostosin.

c.918del, p.(Lys306Asnfs*53)

c.1019G>A, p.(Arg340His) c.1065_1066ins26, p.(Val356Cysfs*12)

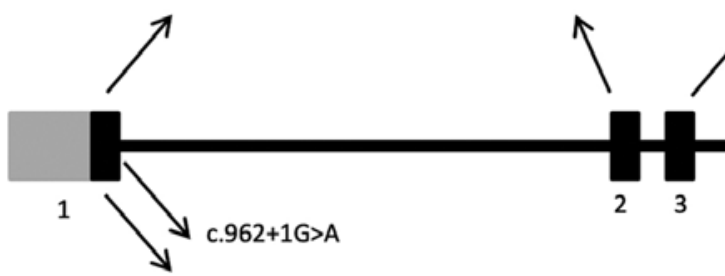

c.96dup, p.(Ser33Glufs*11)

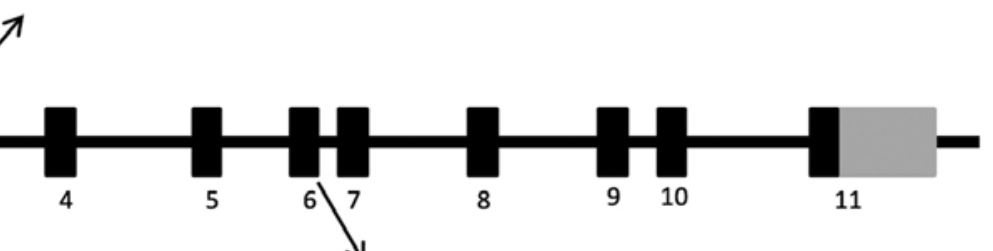

Figure 4. Structure of the EXT1 gene, with the positions of the identified genetic variants indicated. Gray boxes indicate 5' and 3' untranslated region, and black boxes indicate $E X T 1$ exonic region. EXT, exostosin.

whereas the remaining $20 \%$ are frameshift mutations and splice-junction mutations, causing an early termination of translation or partial/complete deletion of the gene and loss of protein function (7).

In the present study, $19 \%(3 / 16)$ of patients with HMO had no point mutations in the coding regions for either EXT1 or EXT2 genes. This may be explained by variants involving large rearrangements such as deletions, duplications, inversions, translocations or somatic mosaicism, that include the EXT1 and EXT2 genes. Deletion of a single or multiple exons were previously detected in $\sim 10 \%$ of all HMO cases $(7,26,27)$. Another study reported a complex rearrangement as causative mechanism of the disease, which involved a $80.7 \mathrm{~kb}$ intronic deletion of EXTlgene and a $68.9-\mathrm{kb}$ duplication proximal of EXT 1 (28). Furthermore, genetic variants in the 5' and 3'UTRs, deep intronic causing variants or in the promoter regions were not determined in the present study. In addition, several studies have reported that $10-15 \%$ of patients with HMO have no mutations in either EXT1 or EXT2 genes (29-31), suggesting that other genes may be involved in the pathogenesis of the disease. Therefore, testing this subgroup of patients by whole exome sequencing or whole genome next generation sequencing will be an attractive approach to identify other possible disease-causing gene(s) $(32,33)$. 

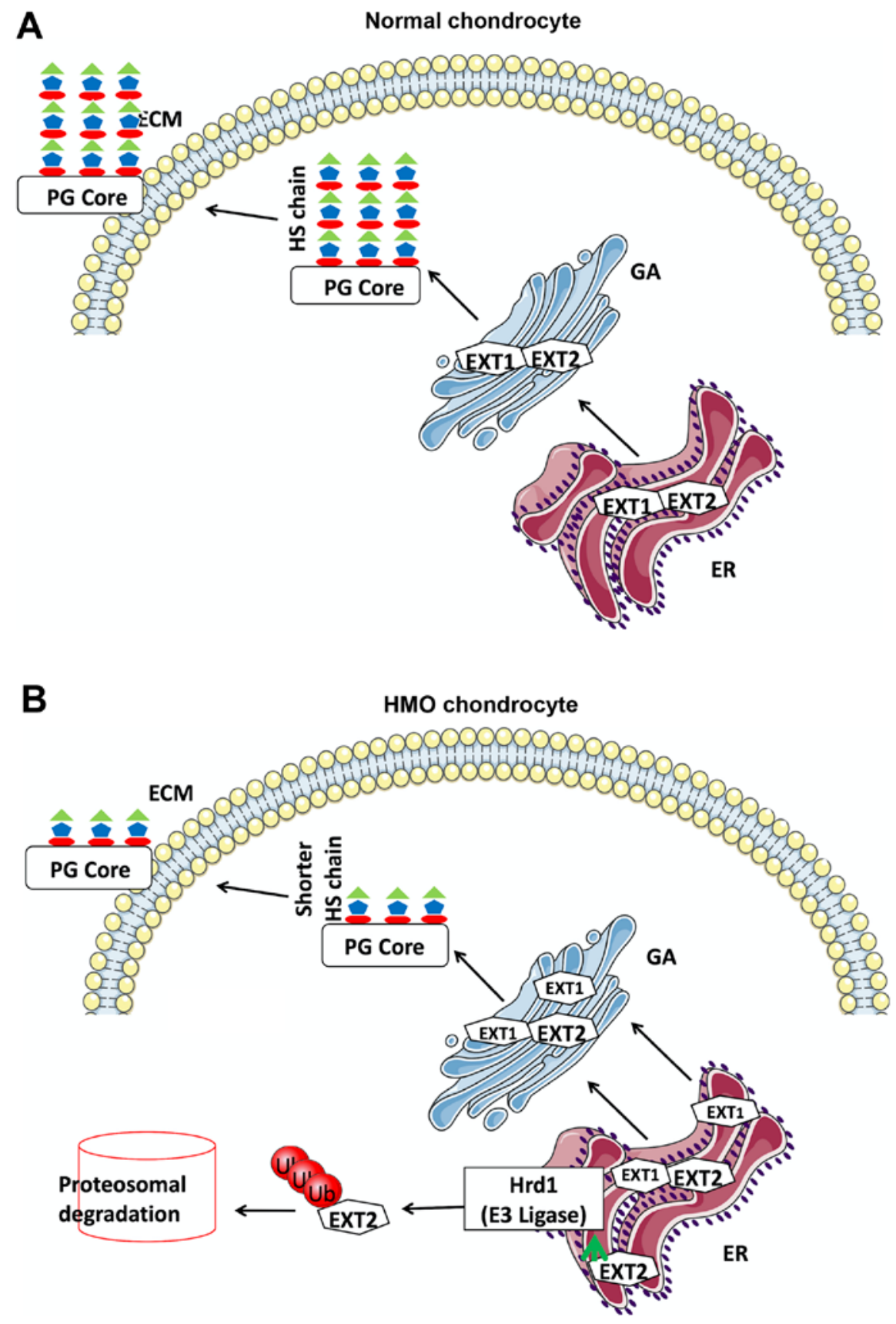

Figure 5. Model of the function of the EXT1/EXT2 complex in HS synthesis in (A) normal chondrocytes and (B) HMO chondrocytes. (A) In normal chondrocytes, EXT1 and EXT2 form a complex, which is involved in the HS synthesis in the Golgi apparatus. The EXT1/EXT2 complex, through its galactosyltransferase activity, aids in the formation of HS proteoglycans HSPGs, which are next exported to the cell exterior. (B) In HMO chondrocytes, decreased levels of EXT1 disrupts the stoichiometry of the EXT1/EXT2 complex, resulting in low HS synthesis and, thus, diminished HSPGs on the cell exterior. Low levels of EXT1 render EXT2 unable to be transported into the Golgi complex, which is instead retained in the ER and thereby targeted for ER-associated protein degradation. EXT2 is shown to be ubiquitinated at Lys 245 and is degraded though ubiquitin-proteasome system. HS, heparan sulfate; HSPGs, HS proteoglycans; EXT, exostosin; HMO; hereditary multiple osteochondromas; ER, endoplasmic reticulum; ECM, extracellular matrix; GA, Golgi apparatus; PG, proteoglycans.

To the best of our knowledge, the majority of phenotype-genotype research studies on HMO did not test for the expression of EXT genes in the resected tumor tissues. Immunohistochemistry studies revealed a decreased expression of EXT1 and/or EXT2 corresponding to the EXT gene mutations status $(3,34,35)$. In the present study, the patients with EXT1 mutations exhibited a decreased expression of EXT1 protein. Surprisingly, the same patients exhibited no expression of EXT2 proteins, although none of them harbored
EXT2 mutations. Previous studies demonstrated that the presence of fully functional EXT1 and EXT2 proteins is required for their correct localization in the Golgi complex $(36,37)$. In addition, the present study proposes a model (Fig. 5), in which mutations in EXT1 result in a truncated product and/or inactive form of the protein that can no longer bind to its EXT2 partner. The very low levels of EXT1, EXT2 and EXT1/2 alter the stoichiometry of the complex and greatly diminish its glycosyltransferase activity. Low levels of EXT1 can no longer 
associate with its requisite partner EXT2 and, thus, EXT2 is retained in the endoplasmic reticulum (ER) and is targeted through the ER-associated protein degradation pathway for degradation. The identification of EXT2 protein as a substrate for the Hrd1 E3 ligase and the identification of the lysine involved in ubiquitin attachment to the protein $(38,39)$ is consistent with the aforementioned model, as explained in Fig. 5.

Mutations in EXT1 and EXT2 are associated with the pathogenesis of HMO; however, the mechanism through which HS synthesis alteration leads to exostoses has yet to be elucidated. Heterozygous EXT1 or EXT2 mutations are common molecular changes identified in $>80 \%$ of the investigated exostoses (40). There remains the question of whether osteochondromas arise via loss of heterozygosity or haploinsufficiency mechanism $(40,41)$. The results of the present study and early biochemical studies $(36,37,42)$ of the EXT1/2 complex suggest that haploinsufficiency for either EXT1 or EXT2 affects the ability of chondrocytes to synthesize HS, as explained in Fig. 5. Although EXT1 and EXT2 are ubiquitously expressed, mutations in these genes are only manifested in chondrocytes, suggesting that chondrocytes require two fully active EXT1 and EXT2 proteins. However, Reijnders et al (41) refuted the haploinsufficiency theory and demonstrated that osteochondromas arise via loss of heterozygosity and inactivation of both alleles (Knudson's two-hit model) (43). Further genetic analysis studies of large cohorts of patients with HMO are required to determine the contribution of LOH and haploinsufficiency in the molecular pathogenesis of the disease.

The main limitation of the present study was the lack of DNA samples from some members of family $\mathrm{C}$ (unaffected individuals I.1 and I.2), and so genetic testing or further investigations for mosaic mutations (pyrosequencing or cloning of the suspected PCR products) could not be conducted.

In conclusion, the present study conducted a phenotype-genotype study of HMO in 16 Jordanian patients from nine families. These patients are representative of an ethnic group in which the genetic background of HMO is infrequently investigated. The majority of these patients were males, diagnosed the age of $\sim 14$ years, and exhibited a mild clinical disease form. Genetic analysis revealed mutations exclusively involving EXT1 gene and none involved EXT2 gene. These mutations were not necessarily associated with a severe clinical disease. Three of the identified mutations were novel. Three patients did not show any mutations for either EXT1 or EXT2 genes. Upon immunohistochemical testing, osteochondroma tissue resected form all patients with EXT1 mutations exhibited decreased expression of EXT1 protein. Surprisingly, EXT2 protein was not detected in these patients although none had EXT2 mutations. Therefore, a model may be suggested that questions the role of EXT2 gene in HMO pathogenies. HMO continues to represent a clinically and genetically heterogenous disease among different ethnic groups. Therefore, further genetic and immunohistochemical studies are required to further elucidate the pathogenesis of HMO. In addition, mutational analysis studies can be helpful in screening for patients with HMO, particularly those who may be at a risk of developing a severe form of the disease, which will have a significant impact on the clinical management and follow up of patients with HMO.

\section{Acknowledgements}

Not applicable.

\section{Funding}

The present study was supported by the Jordan University of Science and Technology (grant no. 20180318).

\section{Availability of data and materials}

Sanger sequencing data are available at https://www.ebi. ac.uk/eva with the following accessions: Project, PRJEB41290; analyses, ERZ1673834. The following links can also be used: https://www.ebi.ac.uk/ena/data/view/PRJEB41290, https://www.ebi.ac.uk/eva/?eva-study=PRJEB41290 and https://wwwdev.ebi.ac.uk/eva/?eva-study=PRJEB41290.

\section{Authors' contributions}

ZM performed the collection and analysis of data, study design, writing and editing of the manuscript. KB performed the data analysis, study design, writing and editing of the manuscript. MA was responsible for the study design and writing of the manuscript. RA performed the data collection, study design and writing of the manuscript. MAA performed the data collection, study design and writing of the manuscript. ABK cotributed to the data collection, study design and writing of the manuscript. KAB performed the data analysis and writing of the manuscript. All authors read and approved the final manuscript.

\section{Ethics approval and consent to participate}

Approval for this research was obtained from the Human Research Ethics Committee and IRB at Jordan University of Science and Technology (approval no. 22/116/2018). All individuals included in this study and/or their legal guardians provided written informed consent for participating in this study.

\section{Patient consent for publication}

All participants and/or their legal guardians provided a written informed consent regarding the publication of case details and any associated images.

\section{Competing interests}

The authors declare that they have no competing interests.

\section{References}

1. D'arienzo A, Andreani L, Sacchetti F, Colangeli S and Capanna R: Hereditary multiple exostoses: Current insights. Orthop Res Rev 11: 199-211, 2019.

2. Hakim DN, Pelly T, Kulendran M and Caris JA: Benign tumours of the bone: A review. J Bone Oncol 4: 37-41, 2015.

3. Bovée JV: Multiple osteochondromas. Orphanet J Rare Dis 3: 3, 2008.

4. Mohaidat ZM, Saleh AA, Al-Omari MH, Obeidat AA and Khasawneh RA: Osteochondroma in Jordanian Patients: Clinical manifestations and management. J Clin Diagnostic Res 12: RC11-RC15, 2018. 
5. Beltrami G, Ristori G, Scoccianti G, Tamburini A and Capanna R: Hereditary multiple exostoses: A review of clinical appearance and metabolic pattern. Clin Cases Miner Bone Metab 13: 110-118, 2016.

6. de Souza AM and Bispo Júnior RZ: Osteochondroma: Ignore or investigate? Rev Bras Ortop 49: 555-564, 2014.

7. Jennes I, Pedrini E, Zuntini M, Mordenti M, Balkassmi S, Asteggiano CG, Casey B, Bakker B, Sangiorgi L and Wuyts W: Multiple osteochondromas: Mutation update and description of the multiple osteochondromas mutation database (MOdb). Hum Mutat 30: 1620-1627, 2009.

8. Zak BM, Crawford BE and Esko JD: Hereditary multiple exostoses and heparan sulfate polymerization. Biochim Biophys Acta 1573: 346-355, 2002.

9. Busse-Wicher M, Wicher KB and Kusche-Gullberg M: The extostosin family: Proteins with many functions. Matrix Biol 35: 25-33, 2014.

10. Mordenti M, Ferrari E, Pedrini E, Fabbri N, Campanacci L, Muselli M and Sangiorgi L: Validation of a new multiple osteochondromas classification through Switching Neural Networks. Am J Med Genet Part A 161A: 556-560, 2013.

11. Fusco C, Nardella G, Fischetto R, Copetti M, Petracca A Annunziata F, Augello B, D'Asdia MC,Petrucci S, Mattina T, et al: Mutational spectrum and clinical signatures in 114 families with hereditary multiple osteochondromas: Insights into molecular properties of selected exostosin variants. Hum Mol Genet 28 2133-2142, 2019.

12. Bali DS, Goldstein JL, Banugaria S, Dai J, Mackey J, Rehder C and Kishnani PS: Predicting cross-reactive immunological material (CRIM) status in Pompe disease using GAA mutations: Lessons learned from 10 years of clinical laboratory testing experience. Am J Med Genet Part C Semin Med Genet 160C: 40-49, 2012.

13. Santos SCL, Rizzo IMPO, Takata RI, Speck-Martins CE Brum JM and Sollaci C: Analysis of mutations in EXT1 and EXT2 in Brazilian patients with multiple osteochondromas. Mol Genet Genomic Med 6: 382-392, 2018.

14. Jäger M, Westhoff B, Portier S, Leube B,Hardt K, Royer-Pokora B, Gossheger G and Krauspe R: Clinical outcome and genotype in patients with hereditary multiple exostoses. J Orthop Res 25: $1541-1551,2007$

15. Porter DE, Lonie L, Fraser M, Dobson-Stone C, Porter JR, Monaco AP and Simpson AH: Severity of disease and risk of malignant change in hereditary multiple exostoses. A genotype-phenotype study. J Bone Joint Surg Br 86: 1041-1046, 2004 .

16. Saglik Y, Altay M, Unal VS, Basarir K and Yildiz Y: Manifestations and management of osteochondromas: A retrospective analysis of 382 patients. Acta Orthop Belg 72: 748-755, 2006.

17. Li Y, Wang J, Wang Z, Tang J and Yu T: A genotype-phenotype study of hereditary multiple exostoses in forty-six Chinese patients. BMC Med Genet 18: 126, 2017.

18. Francannet C, Cohen-Tanugi A, Le Merrer M, Munnich A, Bonaventure J and Legeai-Mallet L: Genotype-phenotype correlation in hereditary multiple exostoses. J Med Genet 38: 430-434, 2001.

19. Delgado MA, Martinez-Domenech G, Sarrión P, Urreizti R, Zecchini L, Robledo HH, Segura F, De Kremer RD, Balcells S, Grinberg D and Asteggiano CG: A broad spectrum of genomic changes in latinamerican patients with EXT1/EXT2-CDG. Sci Rep 4: 6407, 2014

20. Pedrini E, Jennes I, Tremosini M, Milanesi A, Mordenti M, Parra A, Sgariglia F, Zuntini M, Campanacci L, Fabbri N, et al: Genotype-phenotype correlation study in 529 patients with multiple hereditary exostoses: Identification of 'protective' and 'risk' factors. J Bone Joint Surg Am 93: 2294-2302, 2011.

21. Sarrión P, Sangorrin A, Urreizti R, Delgado A, Artuch R, Martorell L, Armstrong J, Anton J, Torner F, Vilaseca MA, et al: Mutations in the EXT1 and EXT2 genes in Spanish patients with multiple osteochondromas. Sci Rep 3: 1346, 2013.

22. Heinritz W, Hüffmeier U, Strenge S, Miterski B, Zweier C, Leinung S, Bohring A, Mitulla B, Peters U and Froster UG: New mutations of EXT1 and EXT2 genes in German patients with multiple osteochondromas. Ann Hum Genet 73(Pt 3): 283-291, 2009.

23. Alvarez C, Tredwell S, De Vera M and Hayden M: The genotype-phenotype correlation of hereditary multiple exostoses. Clin Genet 70: 122-130, 2006.
24. Jennes I, Entius MM, Van Hul E, Parra A, Sangiorgi L and Wuyts W: Mutation screening of EXT1 and EXT2 by denaturing high-performance liquid chromatography, direct sequencing analysis, fluorescence in situ hybridization, and a new multiplex ligation-dependent probe amplification probe set in patients with multiple osteochondromas. J Mol Diagn 10: 85-92, 2008.

25. Cousminer DL, Arkader A, Voight BF, Pacifici M and Grant SFA: Assessing the general population frequency of rare coding variants in the EXT1 and EXT2 genes previously implicated in hereditary multiple exostoses. Bone 92: 196-200, 2016.

26. Vink GR, White SJ, Gabelic S, Hogendoorn PC, Breuning MH and Bakker E: Mutation screening of EXT1 and EXT2 by direct sequence analysis and MLPA in patients with multiple osteochondromas: Splice site mutations and exonic deletions account for more than half of the mutations. Eur J Hum Genet 13: 470-474, 2005.

27. Szuhai K, Jennes I, De Jong D, Bovée JV, Wiweger M, Wuyts W and Hogendoorn PC: Tiling resolution array-CGH shows that somatic mosaic deletion of the EXT gene is causative in EXT gene mutation negative multiple osteochondromas patients. Hum Mutat 32: E2036-E2049, 2011.

28. Waaijer CJ, Winter MG, Reijnders CM, de Jong D, John Ham S, Bovée JV and Szuhai K: Intronic deletion and duplication proximal of the EXT1 gene: A novel causative mechanism for multiple osteochondromas. Genes Chromosomes Cancer 52: 431-436, 2013.

29. Hong G, Guo X, Yan W, Li Q, Zhao H, Ma P and Hu X: Identification of a novel mutation in the EXT1 gene from a patient with multiple osteochondromas by exome sequencing. Mol Med Rep 15: 657-664, 2017.

30. Zhang $\mathrm{H}$, Ping XL, Lee PK, Wu XL, Yao YJ, Zhang MJ, Silvers DN, Ratner D, Malhotra R, Peacocke M and Tsou HC: Role of PTCH and p53 genes in early-onset basal cell carcinoma. Am J Pathol 158: 381-385, 2001.

31. Wu Y, Xing X, Xu S, Ma H, Cao L, Wang S and Luo Y: Novel and recurrent mutations in the EXT1 and EXT2 genes in Chinese kindreds with multiple osteochondromas. J Orthop Res 31: 1492-1499, 2013.

32. Liu H, Wu S, Duan L, Zhu W, Zhang S, Hu X, Jia W, Yang G, Liu C, Li W, et al: Identification of a novel EXT1 mutation in patients with hereditary multiple exostosis by exome sequencing. Oncol Rep 33: 547-552, 2015.

33. Zhang F, Liang J, Guo X, Zhang Y, Wen Y, Li Q, Zhang Z, Ma W, Dai L, Liu $\mathrm{X}$, et al: Exome sequencing and functional analysis identifies a novel mutation in EXT1 gene that causes multiple osteochondromas. PLoS One 8: e72316, 2013.

34. Cheung PK, McCormick C, Crawford BE, Esko JD, Tufaro F and Duncan G: Etiological point mutations in the hereditary multiple exostoses gene EXT1: A functional analysis of heparan sulfate polymerase activity. Am J Hum Genet 69: 55-66, 2001.

35. Hameetman L, David G, Yavas A, White SJ, Taminiau AH, Cleton-Jansen AM, Hogendoorn PC and Bovée JV: Decreased EXT expression and intracellular accumulation of heparan sulphate proteoglycan in osteochondromas and peripheral chondrosarcomas. J Pathol 211: 399-409, 2007.

36. McCormick C, Duncan G, Goutsos KT and Tufaro F: The putative tumor suppressors EXT1 and EXT2 form a stable complex that accumulates in the Golgi apparatus and catalyzes the synthesis of heparan sulfate. Proc Natl Acad Sci USA 97: 668-673, 2000.

37. Kobayashi S, Morimoto K, Shimizu T, Takahashi M, Kurosawa H and Shirasawa T: Association of EXT1 and EXT2, hereditary multiple exostoses gene products, in Golgi apparatus. Biochem Biophys Res Commun 268: 860-867, 2000.

38. Lee KA, Hammerle LP, Andrews PS, Stokes MP, Mustelin T, Silva JC, Black RA and Doedens JR: Ubiquitin ligase substrate identification through quantitative proteomics at both the protein and peptide levels. J Biol Chem 286: 41530-41538, 2011

39. Ernst R, Claessen JH, Mueller B, Sanyal S, Spooner E, van der Veen AG, Kirak O, Schlieker CD, Weihofen WA and Ploegh HL: Enzymatic blockade of the ubiquitin-proteasome pathway. PLoS Biol 8: e1000605, 2011.

40. Hall CR, Cole WG, Haynes R and Hecht JT: Reevaluation of a genetic model for the development of exostosis in hereditary multiple exostosis. Am J Med Genet 112: 1-5, 2002.

41. Reijnders CM, Waaijer CJ, Hamilton A, Buddingh EP, Dijkstra SP, Ham J, Bakker E, Szuhai K, Karperien M, Hogendoorn PC, et al: No haploinsufficiency but loss of heterozygosity for EXT in multiple osteochondromas. Am J Pathol 177: 1946-1957, 2010.

42. Busse M and Kusche-Gullberg M: In vitro polymerization of heparan sulfate backbone by the EXT proteins. J Biol Chem 278: 41333-41337,2003.

43. Knudson AG Jr: Mutation and cancer: Statistical study of retinoblastoma. Proc Natl Acad Sci USA 68: 820-823, 1971.

This work is licensed under a Creative Commons Attribution-NonCommercial-NoDerivatives 4.0 International (CC BY-NC-ND 4.0) License. 